\title{
Toma de decisiones clínicas en atención primaria
}

\author{
Clinical decision making in Primary Care
}

\author{
Antonio Moncada ${ }^{1}$, María Sofía Cuba ${ }^{2}$
}

\section{RESUMEN}

El profesional del primer nivel de atención necesita estar altamente calificado en tomar decisiones médicas con el debido respaldo científico, aplicado a una metodología ordenada y eficiente a la hora de tomar una decisión. De esta manera llegamos a un diagnostico razonado, generando satisfacción en el usuario. A este método lo denominamos toma de decisiones clínicas en atención primaria. Durante el proceso de toma de decisión médica surge la incertidumbre, que en medicina gira en torno a la probabilidad que tiene un paciente de presentar una enfermedad en particular y este puede variar entre 0 y 1 . Según el rango de incertidumbre en el que nos encontremos podemos tomar tres caminos: tratamos al paciente, profundizamos el estudio del caso o simplemente nada. Hay que destacar que existen tres modelos en la toma de decisiones, siendo el más importante, el modelo hipotético deductivo. Finalmente, podemos concluir que no necesariamente tenemos que llegar a un diagnóstico, pero sí debemos llegar a una certeza lo suficientemente alta para actuar.

PALABRAS CLAVE: Atención primaria de salud, decisiones, incertidumbre. (Fuente: DeCS BIREME)

\section{SUMMARY}

The primary care physician needs to be highly qualified in making medical decisions. In making these decisions, he uses all scientific available evidence in an efficient and methodic process, which is called clinical decision-making in primary care. During this process, the uncertainty arises around the probability that a patient has a particular disease ranging from 0 to 1 . According to the range of uncertainty in which we found ourselves, we can take three possible paths: treat the patient, study the patient further, or do nothing. There are three models in clinical decisionmaking; the most important one is the hypothetical-deductive model. Finally, we conclude that physicians not necessarily have to reach a diagnosis, but they must reach a high enough level of certainty to act.

KEYWORDS: Primary health care, decisions, uncertainty. (Source: MeSH NLM)

1 Médico Residente de Medicina Familiar y Comunitaria, Universidad Peruana Cayetano Heredia, RAR-EsSalud, Lima, Perú.

2 Médica Familiar, Magister en Gerencia Social, Docente de la Universidad Peruana Cayetano Heredia, Presidenta de la Sociedad Peruana de Medicina Familiar y Comunitaria (SOPEMFYC), Jefe Médico del Policlínico Juan José Rodriguez Lazo-EsSalud. Lima, Perú. 


\section{INTRODUCCIÓN}

Se define "toma de decisiones" al proceso intelectual que lleva a una elección entre varias posibilidades y que es común a varias situaciones. A partir de este concepto, el proceso de solución de los problemas de salud de individuos enfermos puede ser considerado como un proceso continuo de toma de decisiones médicas, de las cuales depende, invariablemente, el éxito o el fracaso de la gestión del médico (1-3).

Para hacer un diagnóstico, el médico cuenta con sus conocimientos y habilidades clínicas. El profesional del primer nivel de atención necesita estar bien capacitado en tomar decisiones médicas mediante un respaldo científico con una metodología ordenada y eficiente para decidir sobre la base de sus hipótesis diagnósticas, el manejo terapéutico, que conduzca a la solución del problema de salud del paciente. De esta manera llegamos a un diagnostico razonado, simple y costo-efectivo, creando un impacto de satisfacción en el usuario. A este método lo denominamos toma de decisiones clínicas en atención primaria y se considera parte esencial de las competencias que los médicos que laboran en el primer nivel deben practicar.

\section{¿CÓMO TOMAMOS DECISIONES EN ATENCIÓN PRIMARIA?}

Veamos el siguiente ejemplo:

Un paciente varón de 38 años acude a un centro de atención primaria por un dolor tipo punzada en región precordial no desencadenado por el ejercicio y que por momentos se irradia hacia la mandíbula. El médico lo examina y no encuentra alteración cardiovascular alguna. Sospecha de una costocondritis y lo envía a su casa, recomendándole reposo y prescribiendo analgésicos. Horas más tarde los familiares del paciente llevan al paciente con urgencia al centro de atención primaria porque su estado se ha deteriorado mucho. El dolor ha aumentado significativamente, se observa diaforesis, y dolor precordial tipo opresivo irradiado al brazo izquierdo. El médico decide referirlo de inmediato al hospital, donde se realizan exámenes complementarios encontrando elevación de troponinas en sangre y alteraciones en el EKG. Esto, sumado al antecedente de tabaquismo, sedentarismo $y$ diabetes mellitus, lleva a pensar en infarto agudo de miocardio, iniciándose tratamiento especializado.

El diagnóstico diferencial del dolor precordial es amplio e incluye: costocondritis, infarto agudo de miocardio, disección de la aorta, disfagia motora, síntomas de ansiedad, entre otras. El médico debe, ante este panorama, plantear la hipótesis correcta en función de las características presentes o ausentes en el cuadro clínico (dolor precordial tipo opresivo, irradiado a brazo izquierdo, desencadenado por el ejercicio, diaforesis, obesidad, tabaquismo, Diabetes mellitus) y llegar al umbral de confirmación de su diagnóstico y el umbral de exclusión de otras enfermedades que podrían tener repercusiones graves en la salud del paciente (4).

El error en este caso consistió en quedarse con el diagnóstico más frecuente y menos grave por la edad de presentación y características atípicas iniciales con el que se manifestó el dolor precordial, sin tener en cuenta los factores de riesgo como tabaquismo, sedentarismo y antecedente de diabetes mellitus (DM); esto significó pasar por alto otras enfermedades que podrían ser graves, como el infarto agudo de miocardio (IMA). A esto, se le denomina sesgo de representatividad derivado del conocimiento de la probabilidad previa, al no ser un dolor precordial típico, el caso podría corresponder a una costocondritis para los médicos; sin embargo, al tener todos los antecedentes mencionados que elevaban su riesgo cardiovascular, seguramente se hubiera concluido que la probabilidad de un síndrome coronario agudo sobrepasaba el umbral en el que no es necesario hacer más estudios e intervenir.

\section{INCERTIDUMBRE Y PROBABILIDAD EN MEDICINA}

La probabilidad que tiene un paciente de presentar una enfermedad en particular varía entre 0 y $1(0 \%$ a $100 \%$ ) (1). Dentro de este rango, se postula la siguiente pregunta ¿A partir de qué grado de certeza podemos o debemos iniciar una acción contra esta enfermedad? $(4,5)$.

Veamos otro ejemplo:

Un niño de 6 años es llevado por su padre a la consulta. El niño 1 día antes empezó con escalofríos, fiebre y estornudos. En el examen fisico el médico encontró solo congestión nasal. El resto del examen fisico fue normal.

Ante este cuadro, el médico decidió comunicar al padre que el niño presentaba resfrío común y prescribió el tratamiento pertinente, recomendándole que regrese en caso no haber mejoría. En este caso no se consideró indispensable solicitar una radiografía de tórax, menos aún un cultivo de secreción faríngea. Con los pocos argumentos de la anamnesis y del 


\section{CAMPOSDEDECISION}

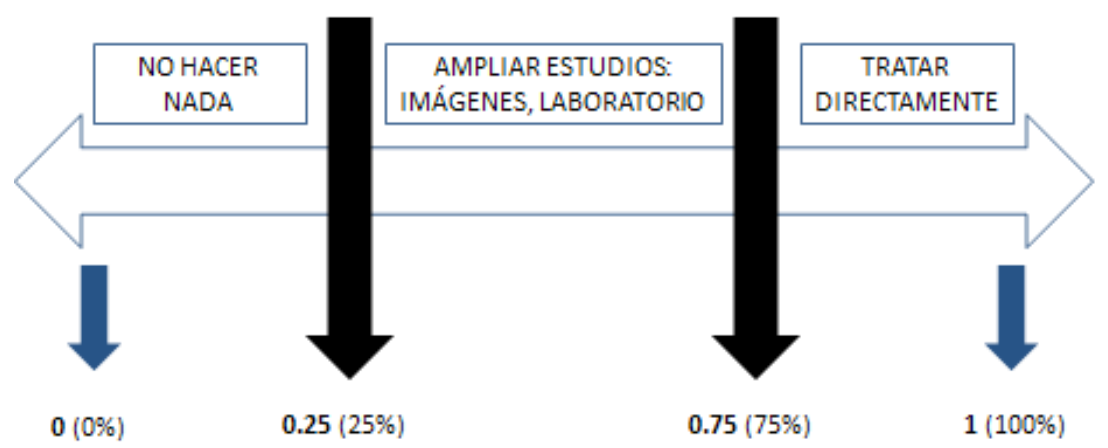

Figura 1. Campos de decisión médica. El criterio de decisión puede encontrarse en cualquier lugar dentro del rango de 0 a 1 ( 0 a 100\%). Mientras más alto está, más seguros debemos estar antes de actuar.

examen clínico, llegó a un nivel de certeza suficiente para poder actuar, sin necesidad de profundizar su investigación diagnóstica. Esto lo podemos graficar en la figura 1.

Van den Ende (6), nos dice: "El criterio de decisión es una balanza entre el riesgo de tratar y no tratar, de solicitar y no solicitar exámenes de laboratorio para profundizar el caso. Conlleva poner en una balanza los beneficios y los riesgos a beneficio del paciente. Mientras más arriba nos situamos al umbral (acercándose a $0 \%$ ), más riesgo corremos de considerar equivocadamente a un buen número de pacientes como personas sanas mientras que en verdad sufren de una patología (falsos negativos) y, por consecuencia, los dejamos sin el tratamiento que necesitan. Esta situación es particularmente nefasta cuando la enfermedad es grave (causa mortalidad o invalidez, por ejemplo, la meningitis bacteriana), cuando es vulnerable por una acción terapéutica (por ejemplo, un antibiótico) o cuando se corre el riesgo de tener graves consecuencias sobre el medio (por ejemplo, una enfermedad epidémica). En cambio, si situamos el umbral muy abajo (cerca de 100\%), más riesgo tenemos de considerar erróneamente a un buen número de personas sanas como enfermas afectadas por cierta patología (falsos positivos), tratándolas innecesariamente".

Por lo visto anteriormente, los médicos en su práctica diaria con los pacientes generan una cantidad limitada de hipótesis. Las hipótesis se confirman o se descartan en un proceso continuo en el cual los conocimientos y la experiencia personal desempeñan un papel importante. Entonces uno puede tomar una de las siguientes decisiones:
1. No pedir estudios ni dar tratamiento.

2. Obtener más información (pedir estudios y decidir sobre la base de los resultados).

3. Tratar directamente sin obtener información adicional $(4,7)$.

\section{MODELOS EN LA TOMA DE DECISIONES}

Sackett (8), enumeró tres estrategias para el diagnóstico clínico: Razonamiento inductivo, hipotético-deductivo y de reconocimiento de patrones.

\section{Modelo RAZONAMIENTO INDUCTIVO.}

Este modelo de razonamiento busca la información sin estar dirigida desde un inicio por alguna hipótesis; es decir se procede a la anamnesis y exploración de aparatos y sistemas que ayudaran a formular una primera hipótesis.

Veamos un ejemplo:

Paciente de 42 años de edad, acude a un centro de atención primaria por inestabilidad moderada, nistagmo unidireccional eimportantes manifestaciones vegetativas,...el médico realiza la anamnesis y examen fisico encontrando un Dix-Hallpike positivo. De esta manera el médico ejecuta su primera hipótesis: vértigo paroxístico posicional benigno.

\section{Modelo HIPOTÉTICO-DEDUCTIVO.}

Este modelo considera que al inicio del proceso se establecen hipótesis basadas en la evidencia disponible, o incluso en la intuición, que después se someten a prueba mediante la exploración y las exámenes complementarios. 
Un ejemplo:

Mujer de 30 años, divorciada, gerente de un empresa y un hijo con parálisis cerebral infantil; manifiesta dolor de cabeza de moderada intensidad, en forma continua en región occipital irradiado hacia los hombros, el cual cede en alguna ocasiones con paracetamol. Al analizar los síntomas y los antecedentes, el médico postula una probable cefalea tensional como primera hipótesis, antes de explorar a la paciente y descartar signos de alarma.

\section{Modelo de RECONOCIMIENTO DE PATRONES.}

Este modelo se plantea como un proceso de mero reconocimiento del cuadro comparándolo con el modelo de la enfermedad que se dispone en la mente, por similitud global, sin ningún tipo de razonamiento.

Ejemplo: Típico caso de paciente de 69 años con dolores articulares en la mano, que se exacerba al despertar por la mañana y mejora con el ejercicio, antiinflamatorios no esteroideos o ambos. El medico diagnostica Artrosis.

\section{LA INCERTIDUMBRE: CARACTERÍSTICA INHERENTE AL PROCESO DE TOMA DE DECISIONES}

La incertidumbre causa sufrimiento psicológico al sentir los profesionales que la medicina no es una ciencia exacta, por eso la ideología médica oficial pretende negar la importancia e incluso la existencia misma de la incertidumbre. Hay que tener en cuenta que las expectativas de la población y la cultura de la profesión, mantienen o generan un impacto social, perceptible como una ciencia exacta. Esta situación se convierte en un círculo vicioso de ansiedad, que dificulta el juicio clínico, predisponiendo al error y puede arrastrar a los profesionales a reacciones disfuncionales.

Un hallazgo clínico de una enfermedad puede presentarse de la siguiente manera:

1. Siempre está presente en los pacientes con la enfermedad; es decir, si el hallazgo está ausente en el paciente, la enfermedad también estará ausente.

2. Nunca está presente en los pacientes que no tiene la enfermedad; por lo tanto, si el hallazgo está presente, la enfermedad también lo está.

Citemos algunos ejemplos de hallazgos clínicos: Maniobra de Dix-Hallpike (+), diagnóstico de vértigo posicional paroxístico benigno (VPPB); cálculos con cristales hexagonales de cistina $(+)$, diagnóstico de Cistinuria.

En medicina este tipo de situaciones se dan de manera excepcional. El médico puede inferir solo a través de evidencias externas imperfectas, y se ve obligado a trabajar en un estado de incertidumbre que se tiene que empezar a "acortar" de alguna manera.

Los datos de la anamnesis, examen físico y datos de laboratorio, pueden dar resultados que no siempre reflejan el verdadero estado del paciente $(5,9,10)$.

\section{¿DE QUÉ MANERA HACEMOS FRENTE A LA INCERTIDUMBRE?}

De modo general, se proponen cuatro respuestas sistemáticas frente a la incertidumbre:

1. Los consensos profesionales y el avance científico proporcionan un apoyo para afrontar la incertidumbre, y sus aportes pueden contribuir a una práctica más apropiada. También facilitan la defensa legal en casos de conflictos judiciales derivados de denuncias de mala praxis.

2. El análisis de decisiones ayuda a pensar de forma más sistemática y a identificar los propios sesgos.

3. Estrategias probabilísticas, aquí el médico debe conocer el potencial informativo de las pruebas más utilizadas y la influencia en sus resultados de la probabilidad pre prueba y post prueba, así como el examen clínico y la valoración del contexto del paciente, de esta manera se hará una utilización lógica del conocimiento científico $(11,12)$.

\section{CONCLUSIÓN}

La meta de una decisión clínica no es necesariamente alcanzar un diagnóstico seguro de una enfermedad, sino más bien llegar a una certeza lo suficientemente alta para actuar y tomar la decisión correcta frente a un paciente y su contexto. Tomar una decisión médica implica ejecutar un razonamiento ordenado respaldado por la evidencia científica, con la intención de tener una aproximación diagnóstica, terapéutica y educativa, mejorando de esta manera la efectividad del médico de atención primaria en beneficio de la salud del paciente; pero esto también nos lleva a asumir riesgos para el paciente, que ha depositado su confianza y su salud en el juicio de su médico, quien cuenta como únicas fortalezas: su capacidad intelectual, sus años de experiencia y el uso 
perspicaz de una buena orientación clínica a la hora de tomar la decisión final.

Por eso, el camino a seguir entre una serie de hipótesis en un paciente en particular puede ser difícil y no sigue reglas fijas, sin embargo, un esquema panorámico que permita priorizar lo grave y tratable, valorando la fuerza de los argumentos a pedir, podría hacer más eficiente el razonamiento clínico y por ende tomar una buena decisión médica.

\section{Correspondencia:}

Antonio David Moncada Catalán

Dirección: Jr. San Martin N ${ }^{\circ}$ 285, Magdalena del Mar Celular: 978372088

Correo: david2024@hotmail.com

\section{REFERENCIAS BIBLIOGRÁFICAS}

1. Núñez F, Leal Z. Interrelación dialéctica de los subsistemas. La toma de decisiones. Ciudad de La Habana: Editorial de Ciencias Médicas; 2000.p.6466.

2. Robbins SP. Fundamentos de comportamiento organizacional. 5ta ed. México: Editorial Prentice; 1998.

3. Robbins SP. Comportamiento organizacional: conceptos, controversias y aplicaciones. México: Editorial Prentice; 1987.p. 561.

4. Rubinstein A. Medicina Familiar y Práctica Ambulatoria. $2^{\circ}$ ed. Buenos Aires: Panamericana; 2006.
5. Sociedad Española de Medicina de Familia y Comunitaria-SEMFYC. Tratado de Medicina de Familia y Comunitaria. $2^{\circ}$ ed. Barcelona: Panamericana; 2012.

6. Van den Ende J, Moreira J, Bisoff Z. Como Tomar Decisiones en Medicina Clínica. $2^{\circ}$ ed. Quito: Salud de Altura; 2008.

7. Forrow L, Wartman A, Brock DW. Science, ethics, and making of clinical decisions. JAMA. 1988; 259:31617.

8. Sackett D, Haynes B, Tugwell P. Clinical epidemiology. A basic science for clinical medicine. Boston/Toronto: Little, Brown and Co; 1985.

9. Kong A, Barnett O, Mosteller F, Youtz C. How medical professionals evaluate expressions of probability. $\mathrm{N}$ Engl J Med. 1986; 315: 740-4.

10. Pauker SG, Kassirer JP. The threshold approach to clinical decision making. N Engl J Med. 1980; 302(20): 1109-17.

11. Hall KH. Reviewing intuitive decision-making and uncertainty: the implications for medical education. Med Educ. 2002; 36(3):216-24.

12. Hunink M, Glasziou P, Siegel J, et al. Decision making in health and medicine. Integrating evidence and values. 1 ed. Cambridge: Cambridge University Press; 2001.

Recibido: 14/05/2013

Aceptado: 17/10/2013 